\title{
The high energy neutrino cross-section in the Standard Model and its uncertainty
}

\author{
Amanda Cooper-Sarkar \\ Particle Physics, University of Oxford, Keble Road, Oxford OX1 3RQ, UK \\ Philipp Mertsch, Subir Sarkar \\ Rudolf Peierls Centre for Theoretical Physics, University of Oxford, \\ 1 Keble Road, Oxford OX1 3NP, UK
}

(Dated: July 25, 2011)

\begin{abstract}
Updated predictions are presented for high energy neutrino and antineutrino charged and neutral current cross-sections within the conventional DGLAP formalism of NLO QCD using modern PDF fits. PDF uncertainties from model assumptions and parametrization bias are considered in addition to the experimental uncertainties. Particular attention is paid to assumptions and biases which could signal the need for extension of the conventional formalism to include effects such as $\ln (1 / x)$ resummation or non-linear effects of high gluon density.
\end{abstract}

Keywords: Deep Inelastic Scattering, Neutrino Physics, High Energy Cosmic Rays.

\section{INTRODUCTION}

Predictions of neutrino cross-sections at high energies have sizeable uncertainties which derive from the uncertainties on the parton distribution functions (PDFs) of the nucleon. In the framework of the quark-parton model, high energy neutrino deep inelastic scattering (DIS) accesses large values of $Q^{2}$, the invariant mass of the exchanged vector boson, and small values of Bjorken $x$, the fraction of the momentum of the incoming nucleon taken by the struck quark. Thus in evaluating uncertainties on high energy neutrino DIS cross-sections it is important to use the most up-to-date information from the experiments at HERA, which have accessed the lowest $x$ and highest $Q^{2}$ scales to date. H1 and ZEUS have now combined the data collected in the years 1994-2000 to give very accurate inclusive cross-sections in the range $6 \times 10^{-7}<x<0.65$ and $0.045<Q^{2}<30000 \mathrm{GeV}^{2}[1]$. We consider modern PDF sets which include these data in order to provide the best benchmark cross-section for experiments searching for high energy cosmic neutrinos such as ANITA [2], IceCube [3], the Pierre Auger Observatory [4] and RICE [5], as well as forthcoming experiments such as ARA [6], ARIANNA [7], JEM-EUSO [8] and LUNASKA [9].

Conventional PDF fits use the next-to-leading-order (NLO) DGLAP formalism [10 13] of QCD to make predictions for DIS cross-sections of leptons on hadrons. At low $x$ it may be necessary to go beyond the DGLAP formalism in order to sum $\ln (1 / x)$ diagrams, as in the BFKL formalism [14 16]. (for recent work see Refs. [17 21]), or to even consider non-linear terms as in the colour glass condensate model [22, 23]. The present paper is concerned with estimating the uncertainties on high energy neutrino cross-sections in the conventional NLO DGLAP formalism. However, the input assumptions of some of the PDF fits are arguably beyond this formalism, and such cases will be highlighted.

Pioneering estimates of high energy neutrino cross-sections were obtained at leading-order [24] using a PDF set (CTEQ4m) which is now well out of date, yet these values continue to be used in analysing current data (e.g. from ANITA [2]). Two of the present authors (CSS) evaluated [26] the cross-sections at NLO using the ZEUS PDFs which included more modern HERA data [25] and using a systematic procedure for estimating PDF uncertainties. More recently the cross-sections have been calculated in Ref. 27] (CTW) using the MSTW2008NLO PDFs, which use HERA data from the same vintage as the ZEUS PDFs and also account for PDF uncertainties. However, neither of these more recent works use the newly combined HERA results which have increased the accuracy of low $x$ data by up to a factor of 3 (see Fig.3 of Ref. [1]). It is the purpose of the present paper to re-evaluate the high energy cross-sections using the most up-to-date PDF sets, with particular emphasis on those which do use these precise, combined HERA data. The calculation is made using PDFs which were evaluated in NLO DGLAP fits, and our calculation of the neutrino structure functions and cross-sections is also made consistently at NLO. The input PDFs we consider treat heavy quarks by using general-mass-variable-flavour number schemes [28, 29], and we have also used such a scheme in our calculation of the structure functions. However, the difference between the use of a general-mass or a zero-mass scheme in the latter part of this procedure is negligible since the neutrino cross-sections are dominated by scattering at high $Q^{2}$. We consider carefully the source of uncertainty on the input PDFs; these derive not only from the experimental uncertainty on the input data but also from model assumptions, and the form of the PDF parameterization. It is important to quantify these uncertainties carefully in order to be able to probe non-perturbative QCD effects at low $x$ [30] and/or new physics beyond the Standard Model [31 34 through a determination of the DIS cross-section using cosmic neutrinos which have energies extending up at least to $\sim 10^{11} \mathrm{GeV}[35]$. 


\section{FORMALISM}

The kinematics of lepton hadron scattering is described in terms of the variables $Q^{2}$, Bjorken $x$, and $y$ which measures the energy transfer between the lepton and hadron systems. The double differential charged current (CC) cross-section for neutrino and antineutrino production on isoscalar nucleon targets is given by [36]

$$
\frac{\mathrm{d}^{2} \sigma(\nu(\bar{\nu}) N)}{\mathrm{d} x \mathrm{~d} Q^{2}}=\frac{G_{\mathrm{F}}^{2} M_{W}^{4}}{4 \pi\left(Q^{2}+M_{W}^{2}\right)^{2} x} \sigma_{\mathrm{r}}(\nu(\bar{\nu}) N),
$$

where the reduced cross-sections $\sigma_{\mathrm{r}}(\nu(\bar{\nu}) N)$ are

$$
\begin{aligned}
& \sigma_{\mathrm{r}}(\nu N)=\left[Y_{+} F_{2}^{\nu}\left(x, Q^{2}\right)-y^{2} F_{\mathrm{L}}^{\nu}\left(x, Q^{2}\right)+Y_{-} x F_{3}^{\nu}\left(x, Q^{2}\right)\right], \\
& \sigma_{\mathrm{r}}(\bar{\nu} N)=\left[Y_{+} F_{2}^{\bar{\nu}}\left(x, Q^{2}\right)-y^{2} F_{\mathrm{L}}^{\bar{\nu}}\left(x, Q^{2}\right)-Y_{-} x F_{3}^{\bar{\nu}}\left(x, Q^{2}\right)\right],
\end{aligned}
$$

and $F_{2}, x F_{3}$ and $F_{\mathrm{L}}$ are related directly to quark momentum distributions, with $Y_{ \pm}=1 \pm(1-y)^{2}$.

The QCD predictions for these structure functions are obtained by solving the DGLAP evolution equations at NLO in the $\overline{\mathrm{MS}}$ scheme with the renormalisation and factorization scales both chosen to be $Q^{2}$. These equations yield the PDFs at all values of $Q^{2}$ provided these distributions have been input as functions of $x$ at some input scale $Q_{0}^{2}$.

In QCD at leading order, the structure function $F_{\mathrm{L}}$ is identically zero, and the structure functions $F_{2}$ and $x F_{3}$ for charged current neutrino interactions on isoscalar targets can be identified with quark distributions as follows:

$$
F_{2}^{\nu}=x(u+d+2 s+2 b+\bar{u}+\bar{d}+2 \bar{c}), \quad x F_{3}^{\nu}=x(u+d+2 s+2 b-\bar{u}-\bar{d}-2 \bar{c}),
$$

and for antineutrino interactions,

$$
F_{2}^{\bar{\nu}}=x(u+d+2 c+\bar{u}+\bar{d}+2 \bar{s}+2 \bar{b}), \quad x F_{3}^{\bar{\nu}}=x(u+d+2 c-\bar{u}-\bar{d}-2 \bar{s}-2 \bar{b}) .
$$

At NLO these expressions must be convoluted with appropriate co-efficient functions in order to obtain the structure functions (and $F_{\mathrm{L}}$ is no longer zero) but these expressions still give us a good idea of the dominant contributions. The contribution of the $b$ quark will be suppressed until scales $\sim m_{t}^{2}$, since the CKM element $V_{t b} \sim 1$. Although the dominant contributions to the CC cross-sections come from $Q^{2} \sim M_{W}^{2} \ll m_{t}^{2}$, this does not mean that the $b$ contribution is always suppressed, because the relevant scale for $t$ production is the virtual boson-nucleon centreof-mass energy, $W^{2} \sim Q^{2} / x$, and the high energy cross-sections are dominated by contributions from very small $x \sim M_{W}^{2} / 2 m_{N} E_{\nu} \approx M_{W}^{2} / s$ (see Fig. 2). This point had been overlooked in earlier work, including our own [26].

The neutral current cross-sections on isoscalar targets are given by expressions similar to Eqs. (1), 22, 3), with the $W$ propagator replaced by the $Z$ propagator, while the leading order expressions for the structure functions given by

$$
\begin{aligned}
F_{2}^{\nu, \bar{\nu}} & =x\left[\frac{\left(a_{u}^{2}+v_{u}^{2}+a_{d}^{2}+v_{d}^{2}\right)}{2}(u+\bar{u}+d+\bar{d})+\left(a_{d}^{2}+v_{d}^{2}\right)(s+b+\bar{s}+\bar{b})+\left(a_{u}^{2}+v_{u}^{2}\right)(c+\bar{c})\right], \\
x F_{3}^{\nu, \bar{\nu}} & =x\left[(u-\bar{u}+d-\bar{d})\left(v_{u} a_{u}+v_{d} a_{d}\right)\right],
\end{aligned}
$$

where $v_{u}, v_{d}, a_{u}, a_{d}$ are the neutral current vector and axial-vector couplings for $u$ - and $d$-type quarks.

\section{PARTON DENSITY FUNCTIONS}

The PDF4LHC group has recently benchmarked modern parton density functions [37]. Since our concern is with high energy neutrino cross-sections, rather than with LHC physics, we focus on PDF sets which make use of the newly combined accurate HERA data [1]. Of all the PDFs considered by the PDF4LHC only HERAPDF1.0 [1]) and NNPDF2.0 [38] used these data. However there has been a subsequent update of the CTEQ6.6 39] PDFs to CT10 [40] which does use these data, while HERAPDF1.0 has recently updated to HERAPDF1.5 [41] using an preliminary combination of HERA data from 2003-2007 as well as the published combined data. We will utilise the CT10 and HERAPDF1.5 PDFs for the present study; we also consider the MSTW2008 PDFs in order to compare with other recent calculations of high energy neutrino cross-sections [27], although we caution that these have not included the most accurate HERA low $x$ data relevant to the present study.

PDFs are generally determined by assuming a parameterization in $x$ which is valid at a starting value of $Q^{2}=Q_{0}^{2}$, where the value of $Q_{0}^{2}$ is chosen to be sufficiently large that perturbative QCD calculations can be applied. The 
form of the parameterization is usually $A x^{B}(1-x)^{C} P(x)$, where $P(x)$ is some smooth function of $x$. Such forms are assumed for the light quarks and the gluon, whereas heavy quarks are generated dyamically from boson-gluon fusion. The PDFs at all other scales $Q^{2}>Q_{0}^{2}$ are calculated by using the DGLAP equations to evolve the parameterized forms in $Q^{2}$. The evolved PDFs are then convoluted with NLO matrix elements to calculate scattering cross-sections for processes of interest. These cross-section predictions are then fitted to deep inelastic data over a broad range of the $x, Q^{2}$ plane (HERA data cover 5 decades in both $x$ and $Q^{2}$ ) in order to determine the parameters of the starting PDF parameterization. ${ }^{1}$ In this way a small number of parameters (10-25, depending on the PDF set) are fitted to a large number (1000-2000) of data points. In this approach one can predict PDFs for $x$ values below those for which data exists because the fitted functional form may be applied for all $x$, although uncertainties will naturally increase outside the fitted region. As we will illustrate, the uncertainty depends mainly on the theoretical prejudice underlying the parameterization at low $x$.

The HERA data form the back-bone of PDF fits and are the only data which extend to the low $x$ region. HERAPDFs use exclusively these data, while MSTW and CT also use older, fixed target data, Drell-Yan data including $W$ and $Z$ production, and Tevatron jet data. Moreover HERAPDFs are unique in using only proton data, so they are free of any assumptions concerning heavy target corrections and deuterium binding corrections [42].

PDFs are presented accounting for the correlated systematic errors of the data as well as the statistical and uncorrelated sources, however, each PDF group has its own approach to the estimation of confidence limits on the uncertainties. A general discussion of approaches to estimating PDF uncertainties is given in Refs. 25, 43] and the approaches used in the PDF sets considered here are reviewed in the PDF4LHC document [37]. PDF uncertainties can also arise from input assumptions made in the PDF fitting. These include the form of the input PDF parameterization at the starting scale for evolution, $Q_{0}^{2}$, the value of $Q_{0}^{2}$ itself, the kinematic cuts made on the data entering the fit, the value of $\alpha_{\mathrm{s}}\left(M_{Z}\right)$, the values of the heavy quark masses — or even the scheme used to account for heavy quark production within the DGLAP fits. The PDFs considered here all account for the heavy quark production using general mass variable flavour number schemes, although the specific schemes differ: MSTW2008 and HERAPDF1.5 use the Thorne-Roberts scheme [28, 29], whereas CT10/CTEQ6.6 use the ACOT scheme [44].

The experimental uncertainties on the PDFs are presented as eigenvector error sets. These eigenvectors represent linear combinations of the PDF parameters which are uncorrelated with each other since they are obtained by diagonalisation of the error matrix of the fitted parameters. Thus uncertainties on the PDFs, and quantities derived from them, can be calculated simply from adding in quadrature the difference between the independent eigenvector sets and the central PDF set. ${ }^{2}$ However the confidence limits represented by these PDF uncertainties are not always set using the conventional tolerances of $\Delta \chi^{2}=1$ for a $68 \%$ c.l., and $\Delta \chi^{2}=2.7$ for a $90 \%$ c.l. Instead both CT(EQ) and MSTW increase the tolerances to account for the marginal inconsistencies of some of the input data sets, and for possible parameterization bias. The exact value of the tolerance is different for each eigenvector (see the individual PDF publications for details). The average tolerance for $90 \%$ c.l. is $\Delta \chi^{2} \sim 5$ for MSTW2008 and $\Delta \chi^{2} \sim 10$ for CTEQ6.6 and CT10. Note that MSTW supply both $68 \%$ and 90\% c.l. sets, whereas CT10/CTEQ6.6 provide only $90 \%$ c.l. sets; since we wish to consider $68 \%$ c.l. uncertainties, we simply scale the CT10 error sets by $(1.64)^{-1}$.

The HERAPDFs use $\Delta \chi^{2}=1$ to set the size of their $68 \%$ c.l. experimental errors. The combination of the HERA data results in a consistent data set with small, well understood systematic errors [1]. The HERAPDF1.0 used NC and $\mathrm{CC} e^{+}$and $e^{-}$cross-section data from the first phase of HERA running 1992-2000 (HERA-I), while the HERAPDF1.5 update uses the preliminary combination of both HERA-I and HERA-II (2003-2007) running. Both of these HERAPDF sets provide not only PDF eigenvector error sets for experimental errors but also PDF variation sets which account for variation of input assumptions: namely variation of the charm and beauty masses, variation of the strangeness fraction in the sea, variation of the $Q^{2}$ cuts applied to the data, variation of the parametrization at $Q_{0}^{2}$ and of the value of $Q_{0}^{2}$ itself. Not all of these sources of uncertainty are specifically considered by the other PDFs.

A potentially important uncertainty is the variation of the minimum $Q^{2}$ cut for data entering the fit. This is set at $Q^{2}>3.5 \mathrm{GeV}^{2}$ for HERAPDF1.5 and at $Q^{2}>2.5 \mathrm{GeV}^{2}$ for CT10 and MSTW2008. Although this cut should be high enough to avoid the non-perturbative region, it may include low $x$ data for which BFKL, or other beyond-DGLAP effects, are already important. Hence we vary this cut to investigate the possible bias thus introduced.

More importantly, HERAPDFs consider a variant of the input parameterization of the gluon at the starting scale which allows the gluon to become negative at low $x, Q^{2}$. At NLO the gluon PDF does not have to be positive, although one might consider that it going negative signals a breakdown of the DGLAP formalism. This form of the gluon is in fact standard in the MSTW2008 parameterization; by contrast, the CT(EQ) analyses do not allow

1 Note that not all parameters are fitted: the normalisations of the gluon and the $u$ and $d$ valence quarks are fixed by the momentum and number sum rules respectively while some other parameters are fixed by model assumptions; the individual publications of the PDF groups should be consulted for details [37].

2 We have used Eq.(19) of Ref. [45], which allows for asymmetry of the positive and negative excursions along the same eigenvector 
such negative gluon variants. However measurable quantities such as the longitudinal structure function $F_{L}$, which is closely related to the gluon at small $x$, must be positive. Therefore we check whether this is the case when a negative gluon PDF is encountered.

A further uncertainty concerns the choice of the value of $\alpha_{\mathrm{S}}\left(M_{Z}\right)$ because the shape of the low $x$ gluon and the value of $\alpha_{\mathrm{s}}\left(M_{Z}\right)$ are correlated through the DGLAP equations. All the PDF sets considered have been determined with a fixed value of $\alpha_{\mathrm{S}}\left(M_{Z}\right)-0.120$ for MSTW2008, 0.118 for CT/CTEQ and 0.1176 for HERAPDF1.5. However PDF sets with a range of fixed values of $\alpha_{\mathrm{s}}\left(M_{Z}^{2}\right)$ are also supplied for all these PDFs. The uncertainty on the PDFs due to $\alpha_{\mathrm{s}}\left(M_{Z}\right)$ can be obtained by varying the value of $\alpha_{\mathrm{s}}\left(M_{Z}\right)$ by \pm 0.0012 at $68 \%$ c.l. [46].

The PDFs discussed above are calculated at NLO in the DGLAP formalism and when they are used to make predictions of the neutrino DIS cross-sections the calculations should naturally be performed at a consistent order as we have done. We have also checked the impact of the use of NNLO PDFs and evolution for HERAPDF1.5 and found it to be negligible.

\section{TECHNICAL DETAILS}

The calculation of the CC and NC cross-sections in NLO has been performed using DISPred [47]. QCDNUM [48] as included in LHAPDF is used for the evaluation of the structure functions $F_{2}, F_{L}$ and $x F_{3}$. The numerical integration of the differential cross-section is performed using the VEGAS Monte Carlo integration routine from the GSL library [49], reducing the integration error to less than $1 \%$.

The most up-to-date PDFs in the LHAPDF [50] format are given in form of grids with a limited kinematic range. For example, the grid extends down to $10^{-8}$ in $x$ for the CT10 and HERAPDF1.5 sets and down to only $10^{-6}$ for the MSTW2008 set. Below this range the PDFs 'freeze', i.e. they take the value at the lower grid boundary. However, for very high energies the total cross-section has significant contributions from lower $x$ (see also Fig. 2) such that the cross-section calculated using the LHAPDF sets will be too small. Therefore, we have used implementations for the PDFs provided by the CTEQ [51] and MSTW [52] groups which allow an extrapolation beyond the grid. Of course, such an extrapolation using e.g. polynomials is not necessarily physical but in the absence of a grid that extends to low enough values of $x$ or the parametric form of the PDFs, this is the only viable alternative. We have checked the agreement between the cross-sections calculated with 'freezing' and with the extrapolations and the difference is more than $50 \%$ for MSTW2008 but only $3 \%$ for CT10 at $E_{\nu}=5 \times 10^{11} \mathrm{GeV}$.

\section{RESULTS}

Figure 1 shows the predicted sea and gluon distributions from the HERAPDF1.5 fit and their fractional uncertainties, at various $Q^{2}$ values. This shows the importance of the low $x$ contribution and illustrates that the PDF uncertainties are largest at low $Q^{2}$ and at low $x$. PDF uncertainties are also large at very high $x$ but this kinematic region is not important for scattering at high neutrino energies.

This is seen from Figure 2 which indicates the kinematic regions in the $x, Q^{2}$ plane which contribute to the neutrino cross-sections for two representative values of the neutrino energy: $s=10^{8} \mathrm{GeV}^{2}\left(\Rightarrow E_{\nu}=5.3 \times 10^{7} \mathrm{GeV}\right)$ in the left panel and $s=10^{10} \mathrm{GeV}^{2}\left(\Rightarrow E_{\nu}=5.3 \times 10^{9} \mathrm{GeV}\right)$ in the right panel. (We do not show the antineutrino cross-sections separately because these are very close to the neutrino cross-sections at high energy.) One can see that the dominant contributions come from $500 \lesssim Q^{2} \lesssim 50000 \mathrm{GeV}^{2}$ and $10^{-6} \lesssim x \lesssim 10^{-2}$ for the lower neutrino energy, and $10^{-8} \lesssim x \lesssim 10^{-4}$, for the higher neutrino energy. Thus although PDF uncertainties are large at low $x$ and low $Q^{2}$, we see that the dominant contributions to the cross-section do not come from the kinematic regions of greatest uncertainty for the PDFs.

Figure 3 compares the gluon PDF and its uncertainty at $Q^{2}=10000 \mathrm{GeV}^{2}$ for the three PDFs which we consider. This value of $Q^{2}$ is in the middle of the range which contributes significantly to the neutrino cross-sections. We see that the central values of the gluon PDFs are all very similar, whereas the uncertainty estimates differ. The CT10 and HERAPDF1.5 uncertainties are actually very similar if we leave out member 52 from the CT10 error set. This error set was introduced into the CT10 analysis to allow for a larger uncertainty at low $x$ [53]. Previous CTEQ analyses such as CTEQ6.6 39] do not have such an extreme error set — see left panel of Fig. 4, The problem with such an ad hoc introduction of a steeply increasing gluon PDF is that at low $x$ it leads to a very strong rise of the neutrino cross-section which seems unphysical (see later discussion).

The larger error band of MSTW2008 is partly due to the fact that it does not include the most up to date HERA data, which have significantly reduced errors at low $x$. However the more striking difference between MSTW2008 and both HERAPDF1.5 and CT10 is the downward divergence of its error band which is due to the gluon becoming negative at low $x, Q^{2}$. This is best understood by reference to the right panel of Fig 4 which shows HERAPDF1.5 both 

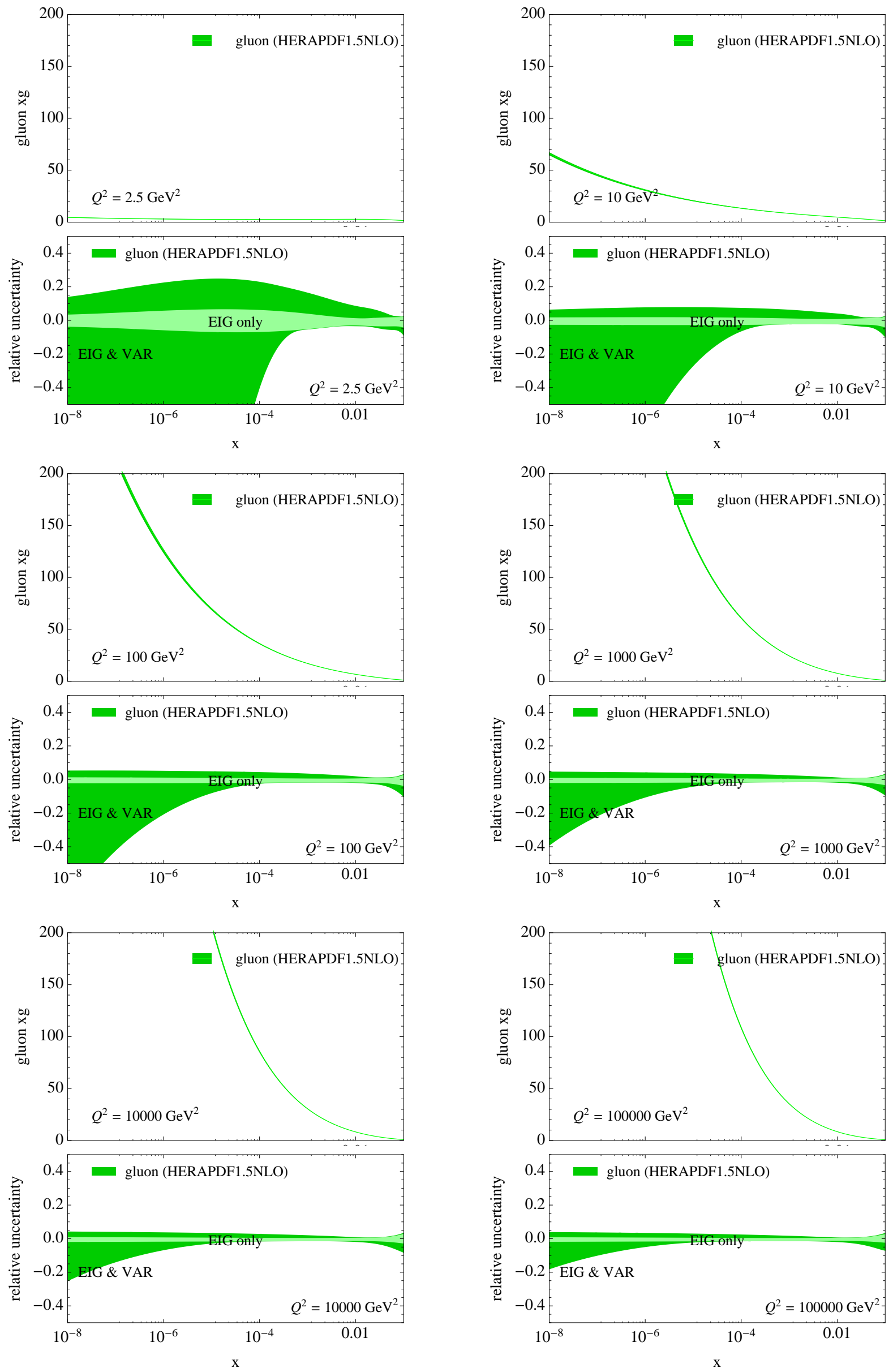

FIG. 1: The HERAPDF1.5 gluon PDFs and their fractional uncertainties — from the experimental errors only (EIG), and from both experimental errors as well as model and parameter variations (EIG \& VAR) - at various values of $Q^{2}$. 

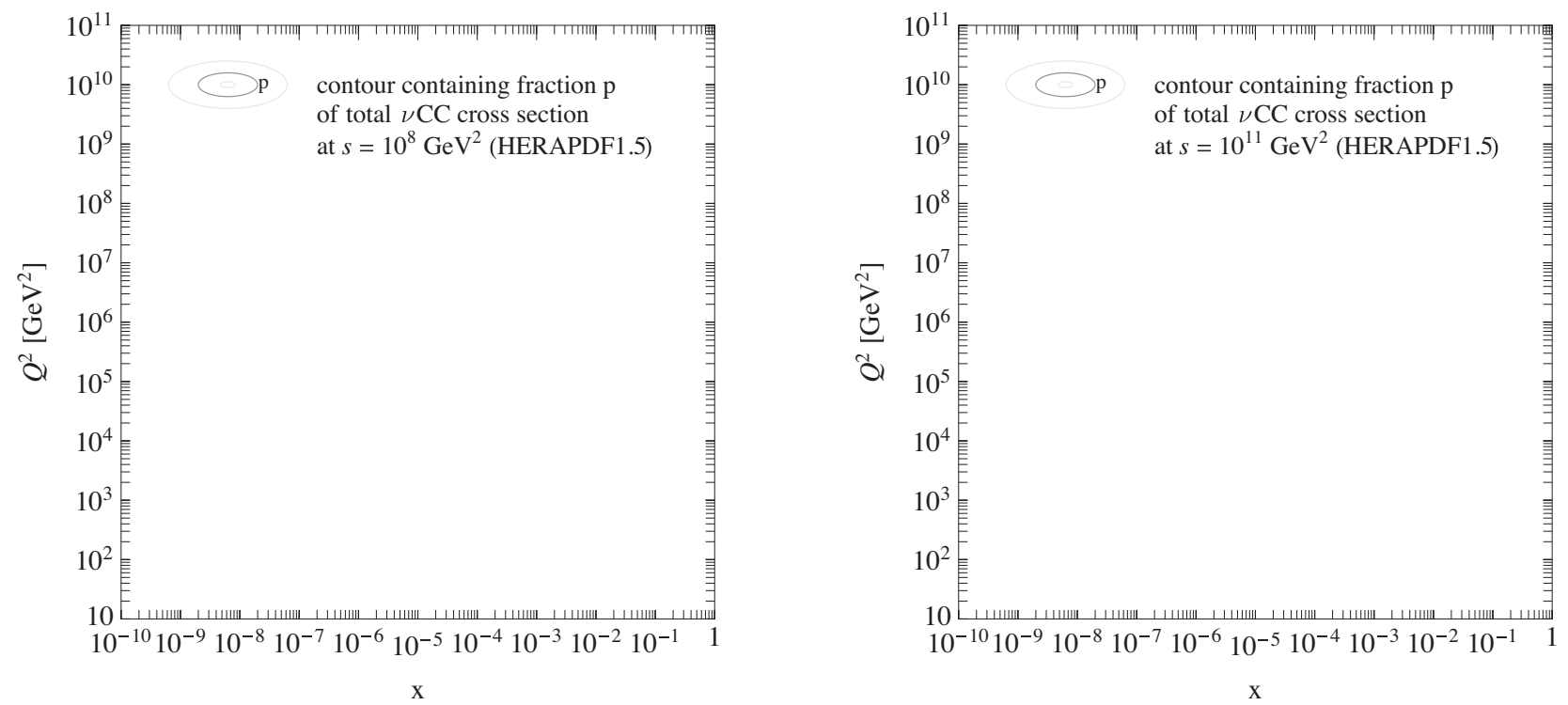

FIG. 2: Kinematic regions of the $x, Q^{2}$ plane and their contribution to the total neutrino cross-section using HERAPDF1.5 for two different values of $s$. The labels show the relative contribution to the total cross-section contained within each contour.
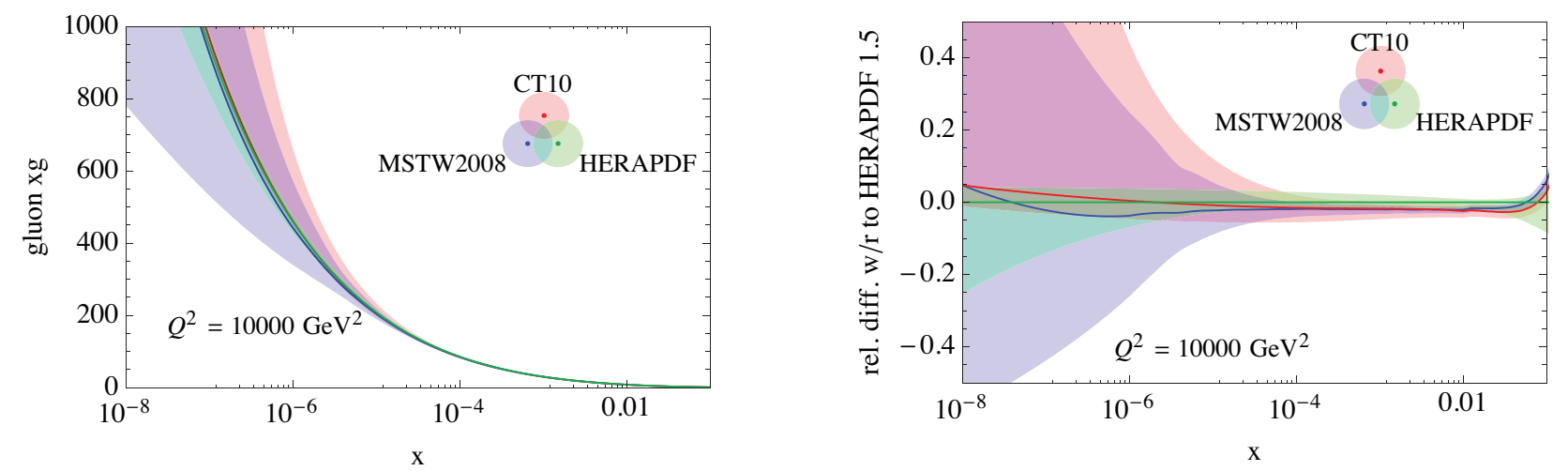

FIG. 3: Left panel: Gluon structure function at $Q^{2}=10^{4} \mathrm{GeV}^{2}$ for the three PDF sets used. Right panel. The relative deviations and uncertainties (at $68 \%$ c.l.) with respect to the central value of HERAPDF1.5. The uncertainty bands are shown with member 9 for HERAPDF1.5 and member 52 for CT10.

with and without member 9 (the variant which allows the gluon to become negative at low $x, Q^{2}$ ). However Fig. 5 shows that while the longitudinal structure function $F_{L}$ is always positive for HERAPDF1.5, it becomes negative, hence unphysical, for some of the MSTW2008 PDF error sets.

The total neutrino cross-sections are now obtained by integrating the predicted double differential cross-section $\mathrm{d}^{2} \sigma / \mathrm{d} x \mathrm{~d} y$ with no cuts on either kinematic variable. Fig. 6] and 7 show the NC and CC neutrino cross-sections as a function of $E_{\nu}$ as evaluated from the HERAPDF1.5 and CT10 PDFs respectively. The PDF uncertainties of these predictions are shown relative to the central values underneath each plot.

It is clear already that the variation of input assumptions considered by the HERAPDF1.5 - apart from the negative gluon variation - do not increase the error band very significantly. Thus the other parameterization variations and the input values of the charm and beauty mass and the fraction of strangeness in the sea are not important for the predictions of neutrino cross-sections. However it is interesting to consider the variation due to the choice of the minimum $Q^{2}$ for data entering the fit. Decreasing this cut to $Q^{2}>2.5 \mathrm{GeV}^{2}$ has negligible effect but the effect of increasing the cut to $Q^{2}>5 \mathrm{GeV}^{2}$ is just about visible in Fig. 6. This larger $Q^{2}$ cut also cuts out data at $x<10^{-4}$ - the kinematic region where there are already some hints of beyond-DGLAP behaviour such as BFKL or non-linear effects. This results in a steeper low $x$ gluon and we can see this in the marginally enhanced neutrino cross-section.

Another potentially important effect comes from the variation of $\alpha_{\mathrm{s}}\left(M_{Z}\right)$ which is correlated to the gluon PDF such that lower values of $\alpha_{\mathrm{s}}\left(M_{Z}\right)$ result in a steeper low $x$ gluon. We evaluate this by considering a $68 \%$ c.l. variation of 

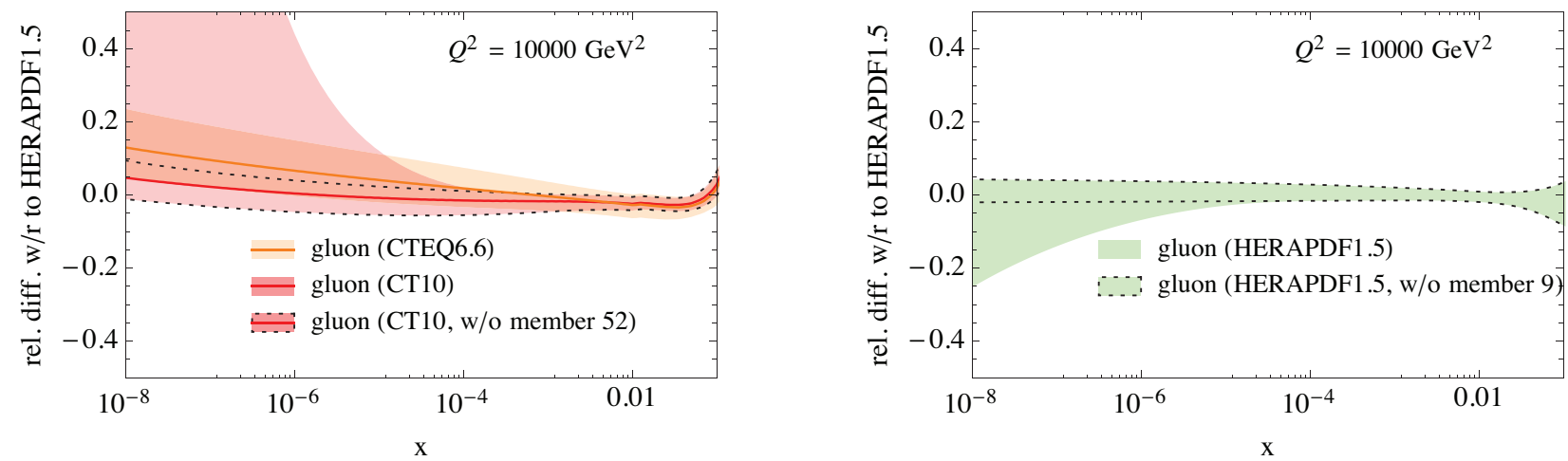

FIG. 4: Uncertainties (at $68 \%$ c.l.) with respect to the central value of HERAPDF1.5 at $Q^{2}=10^{4} \mathrm{GeV}^{2}$. Left panel: CT10 with and without member 52 (CTEQ6.6 shown for comparison). Right panel: HERAPDF1.5 with and without member 9.
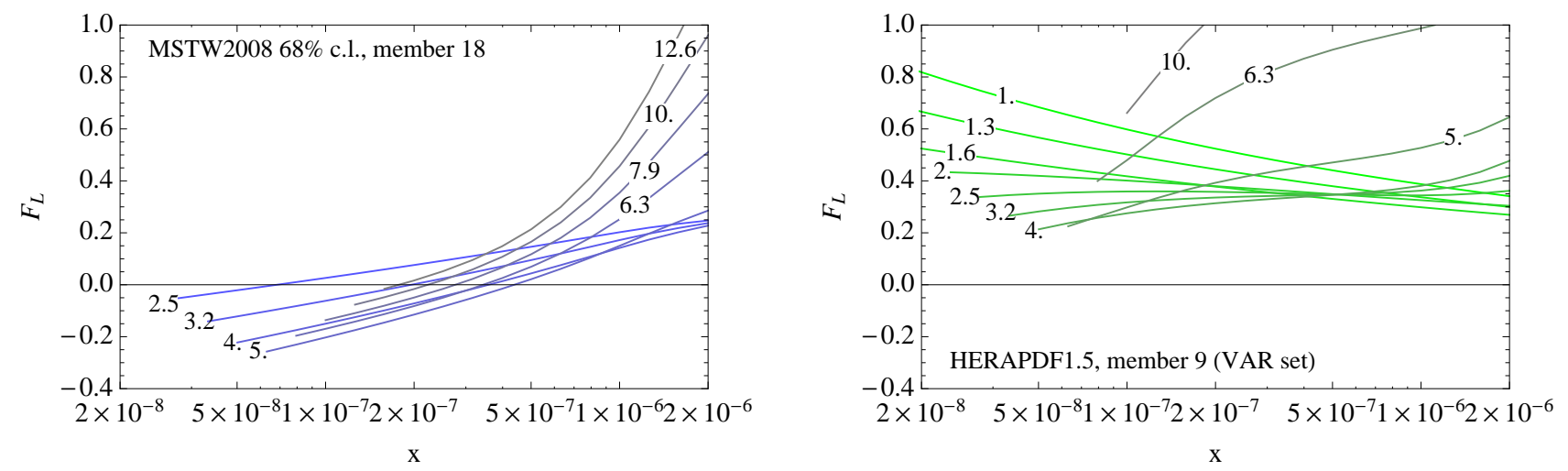

FIG. 5: Left panel: The longitudinal structure function $F_{L}$ calculated from member 18 of MSTW2008nlo68cl for $s=10^{8} \mathrm{GeV} 2$ and different values of $Q^{2}$ (labelled in units of $\mathrm{GeV}^{2}$ ). Right panel: The same for member 9 (VAR set) of HERAPDF1.5.

$\Delta \alpha_{\mathrm{s}}\left(M_{Z}\right) \pm 0.0012$ from its central value. The slight enhancement in the neutrino DIS cross-section is so small that it is not noticeable on the scale of Figs. 6 and 7 hence we have not attempted to show it.

In Figs 8 (top panels) we compare the $\mathrm{NC}$ and $\mathrm{CC}$ cross-sections, along with their total uncertainties (including that coming from the variation of $\alpha_{\mathrm{S}}\left(M_{Z}\right)$ ), as predicted by HERAPDF1.5 and CT10. The MSTW2008 central prediction is also included for comparison. In Fig 8 (bottom panels) we emphasize the small differences in the central values of the PDFs and their relative uncertainties. In order to highlight the effect of the extreme members of HERAPDF1.5 and CT10 in Figs 9, we show these plots without member 9 of the HERAPDF15 variations (which allows for the gluon to become negative at low $x$ and $Q^{2}$ ) and without member 52 for CT10 (the cross-section for which rises $\propto E_{\nu}^{0.7}$ whereas for the central member it rises $\propto E_{\nu}^{0.3}$ ). However any power-law rise in the cross-section will eventually violate the Froissart bound, which requires the rise to be no faster than $\log ^{2} s$ [54]. This should result in a reduction of the cross-section at high energies, by a factor of $\sim 2$ at $E_{\nu}=10^{12} \mathrm{GeV}$ [55] and perhaps even more [56].

There are small differences from the previous work of CSS [26] and CTW [27]. In Fig. 10] we compare our calculation using MSTW2008 PDFs to that of CTW. We find that we can reproduce their results well only if we use the MSTW2008 NLO PDFs together with an leading-order treatment of the coefficient functions, rather than with a consistent NLO approach. In Fig 11] we compare our calculation using HERAPDF1.5 to that of CSS [26]. The predictions for both the central values and the uncertainties of the neutrino NC cross-section are quite close. This is also true for the CC cross-section at low $E_{\nu}$, however above $10^{4} \mathrm{GeV}$ the difference increases since in the present work we have included the contribution of the $b$ quark which was missed out in our earlier work. Accordingly the bounds derived on the cosmogenic neutrino flux using the CSS cross-sections [3, 4] are conservative, being $\sim 20 \%$ too high.

In Fig. 12 we compare our results for HERAPDF1.5 to the cross-sections used in the neutrino event generator ANIS [57] which is based on CTEQ5D. Note that at energies below a TeV (which is the most important energy range for neutrino telescopes like IceCube [58]) there is a $\sim 10 \%$ discrepancy. We also compare the CC cross-section for HERAPDF1.5 to its value in the GENIE low energy neutrino event generator [59] at around $100 \mathrm{GeV}$, finding the match to be consistent within errors. 

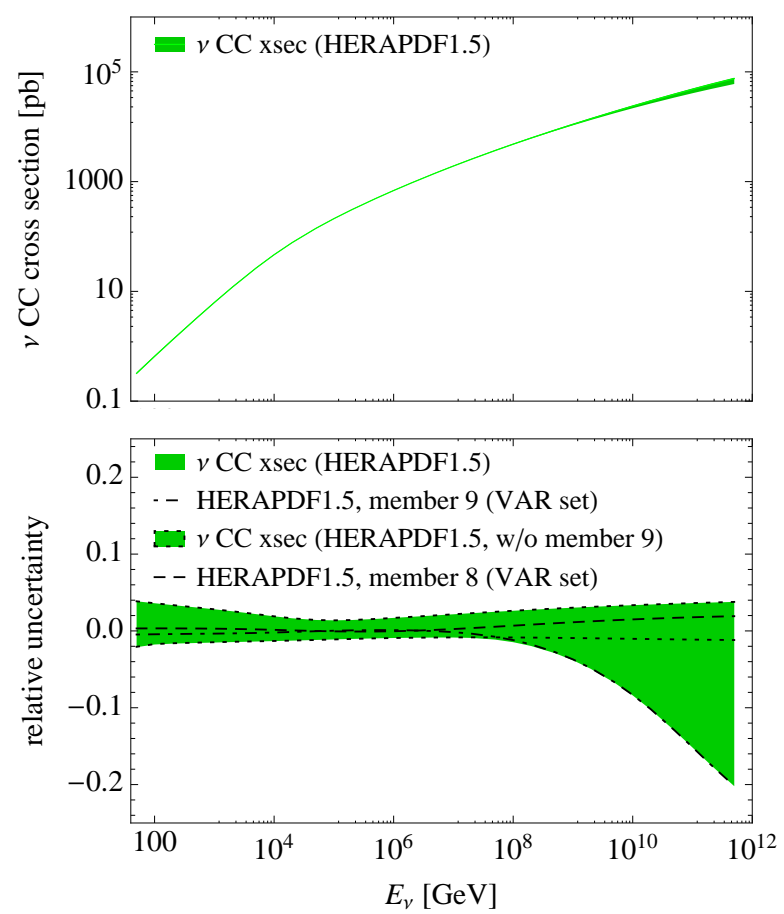
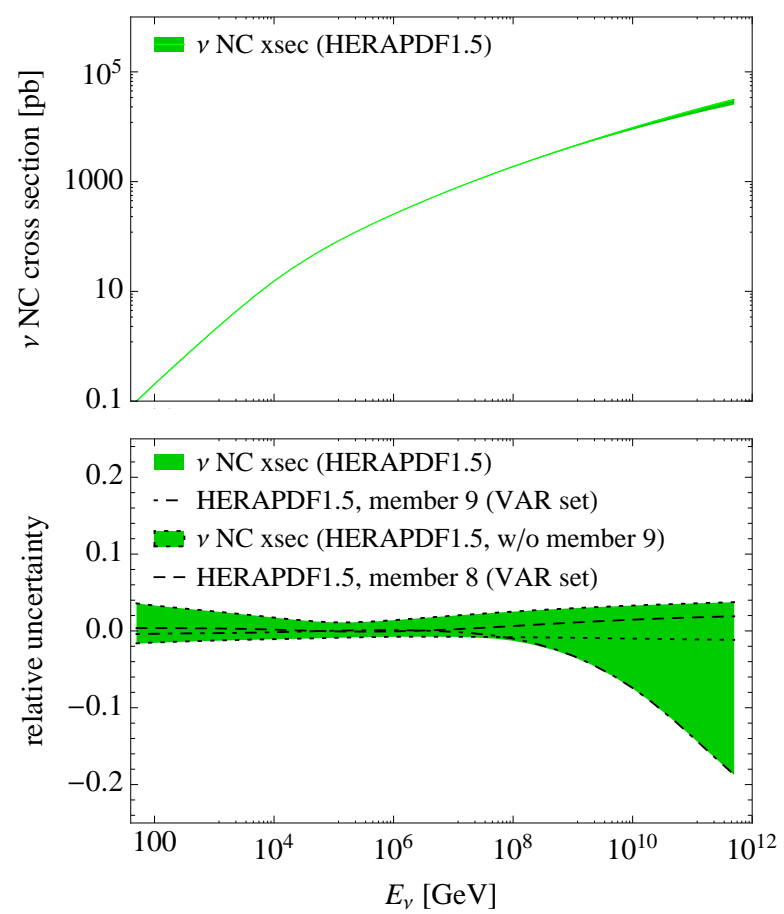

FIG. 6: Neutrino DIS cross-section for CC and NC scattering as predicted by the HERAPDF1.5. Total relative PDF uncertainties including experimental errors as well as model and parameter variations (EIG \& VAR) are shown beneath each plot, both with and without member 9. The marginal effect of increasing the $Q^{2}$ cut from 2.5 to $5 \mathrm{GeV}^{2}$ is also shown (member 8 ).
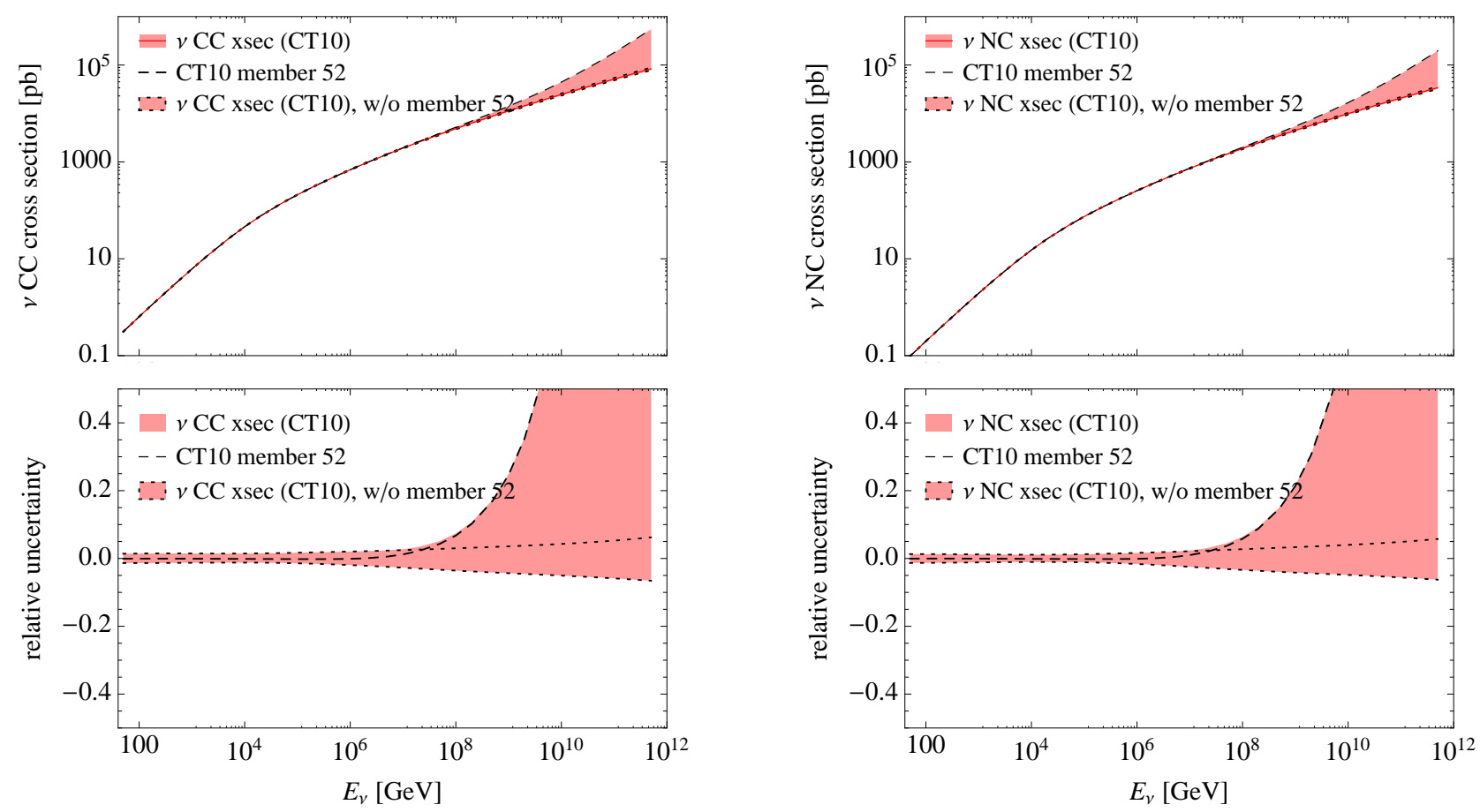

FIG. 7: Neutrino DIS cross-section for CC and NC scattering as predicted by the CT10 PDF. Relative PDF uncertainties from experimental errors only (EIG), and also including parameter uncertainties (EIG \& VAR) are shown beneath each plot, both with and without member 52 . 

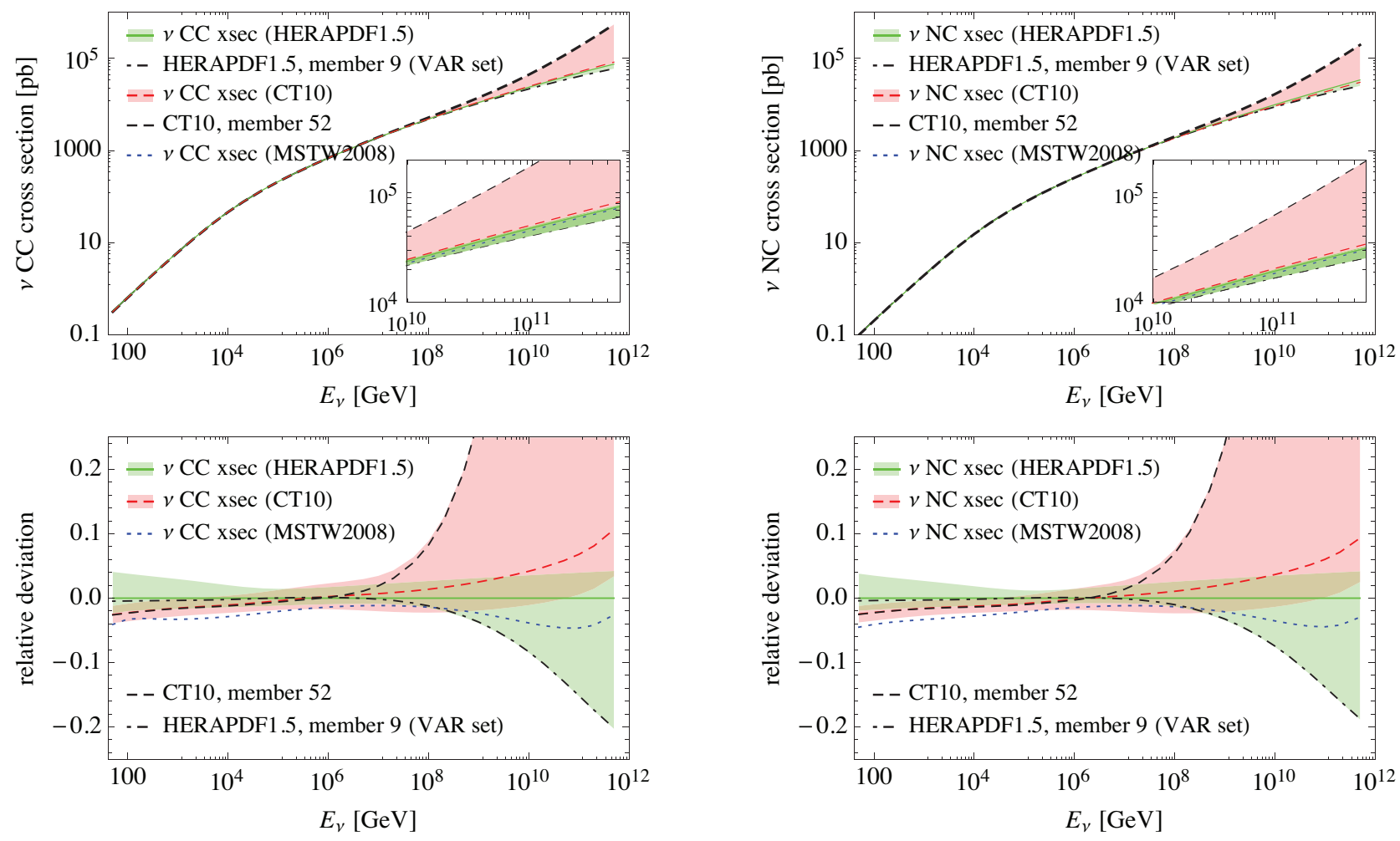

FIG. 8: Comparison of the total cross-section (top panels) and uncertainties (bottom panels) for CC and NC scattering as predicted by the HERAPDF1.5, CT10 and MSTW2008 (central member only) PDF sets. The cross-sections and deviations for member 9 of HERAPDF1.5 and member 52 of CT10 are indicated by the dashed and dot-dashed lines, respectively.
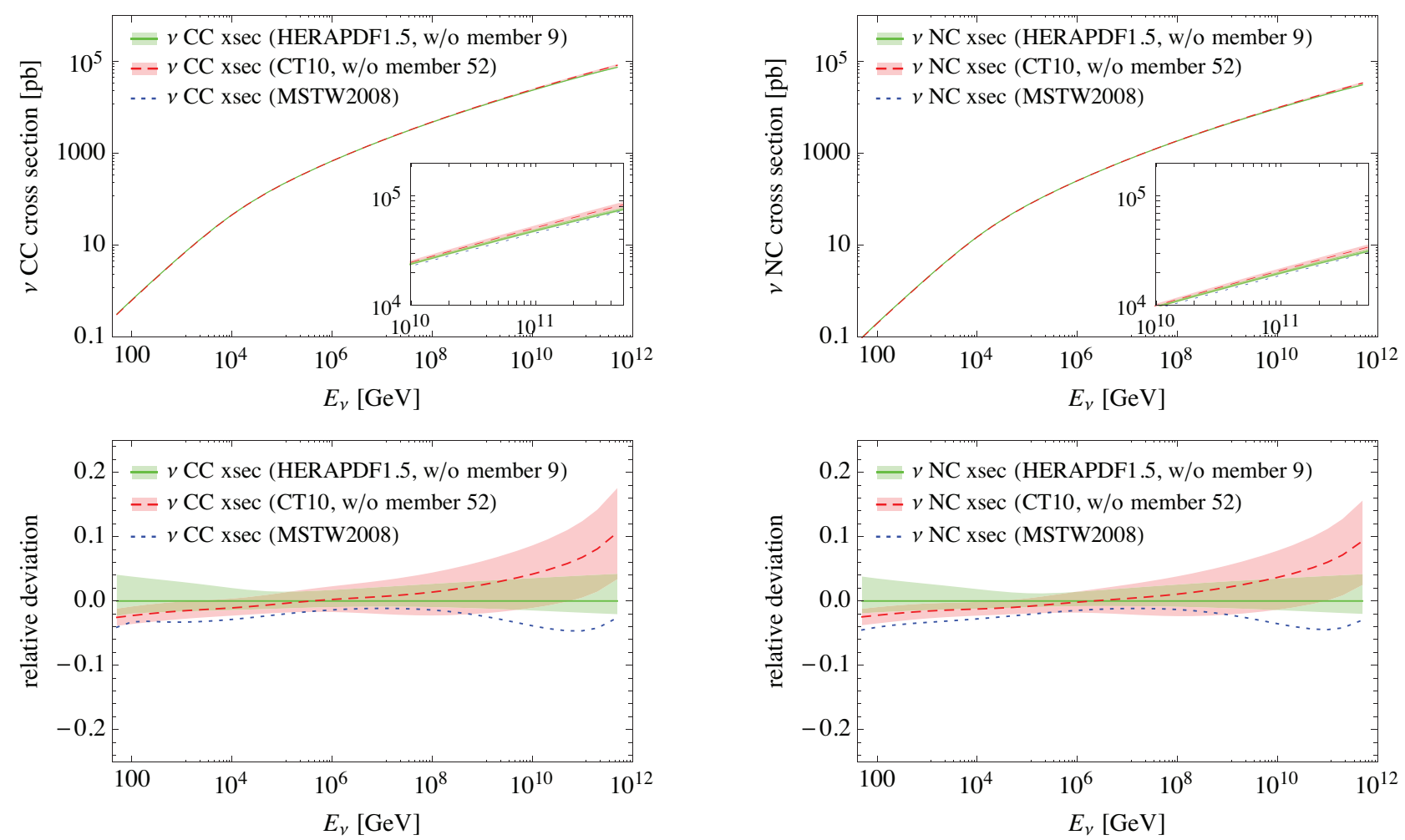

FIG. 9: Same as Fig. 8 but excluding member 9 of the HERAPDF1.5 set and member 52 of the CT10 set. 

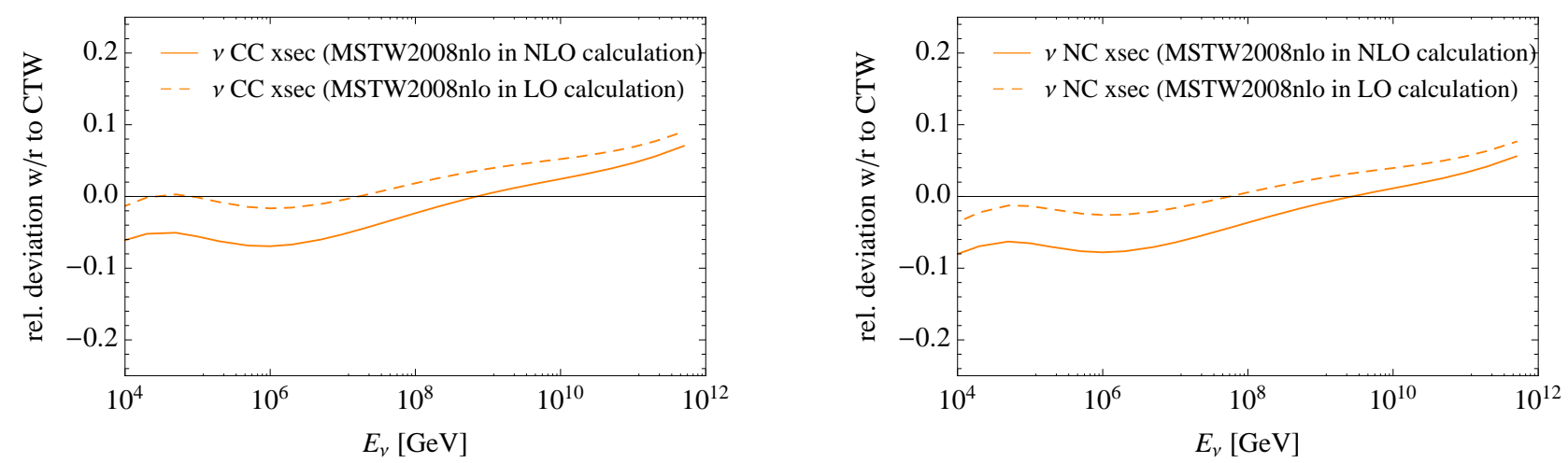

FIG. 10: Our results for the neutrino DIS CC and NC cross-section using the MSTW2008 central member, relative to the results of CTW 27]. The calculation is done consistenly in NLO as well as, for illustration, using a LO code.
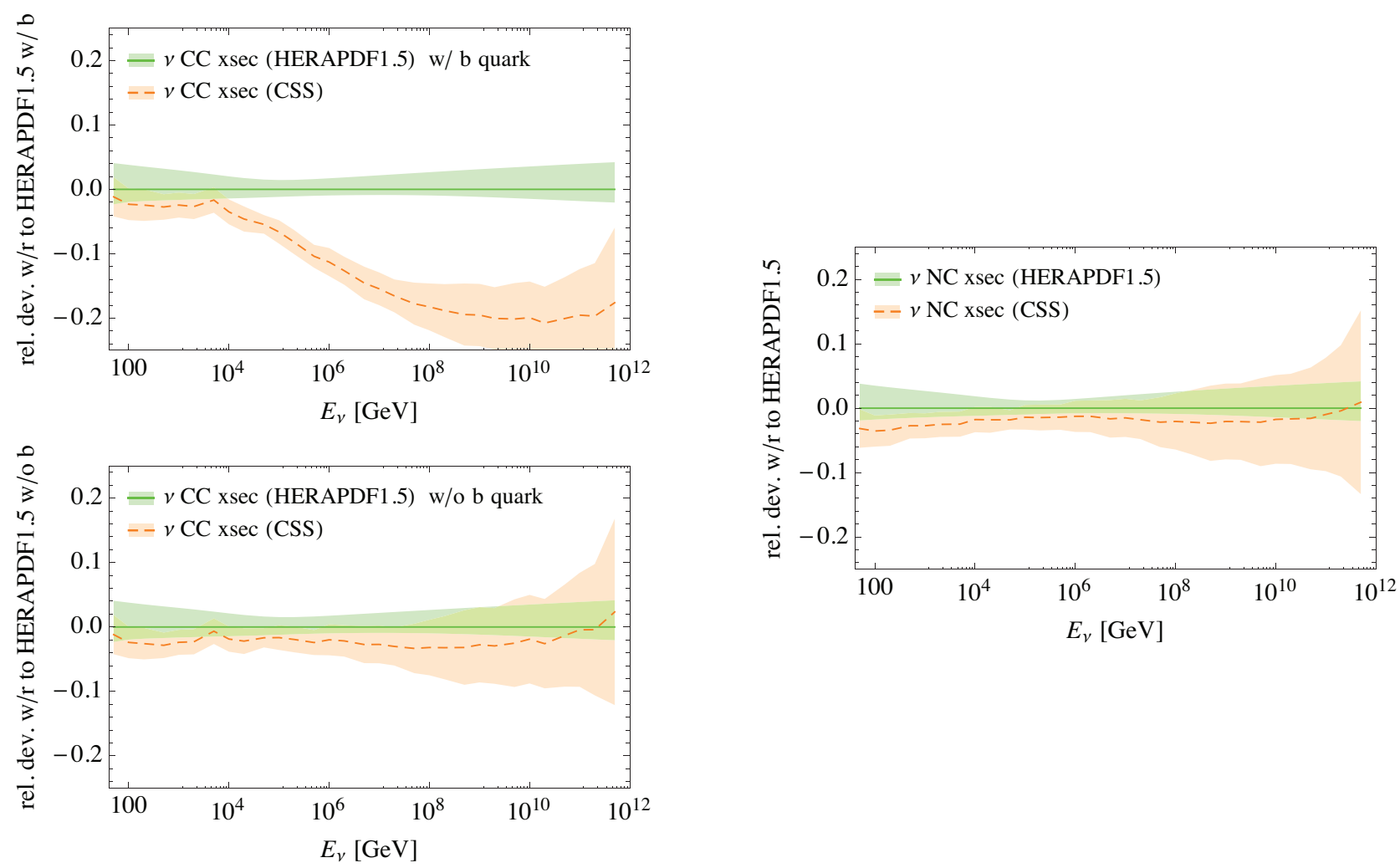

FIG. 11: The relative deviation of the cross-section calculated by CSS 26] from our result for the HERAPDF1.5 central member. For the CC cross-section (left) we compare to HERAPDF1.5 with (upper panel) and without (lower panel) the $b$-quark contribution. For NC scattering (right) the $b$-quark was included already by CSS [26] and the agreement is excellent.

\section{CONCLUSIONS}

We find that the predictions of high energy neutrino DIS cross-sections from the central values of HERAPDF1.5, CT10 and MSTW2008 PDFs are very similar. However the predictions for the uncertainties (deriving from the uncertainties on the input PDFs) differ quite strongly. In fact PDF uncertainties derive from the input assumptions as well as from the input experimental data. If we exclude error sets which either lead to too steep a rise in the crosssection, or allow the low $x$ gluon to be negative at low $Q^{2}$, then we find that the uncertainty estimates of HERAPDF1.5 and CT10 - both of which use the most up-to-date, accurate HERA data - are remarkably consistent.

Our results for the high energy neutrino and antineutrino CC and NC DIS cross-sections and their uncertainties 

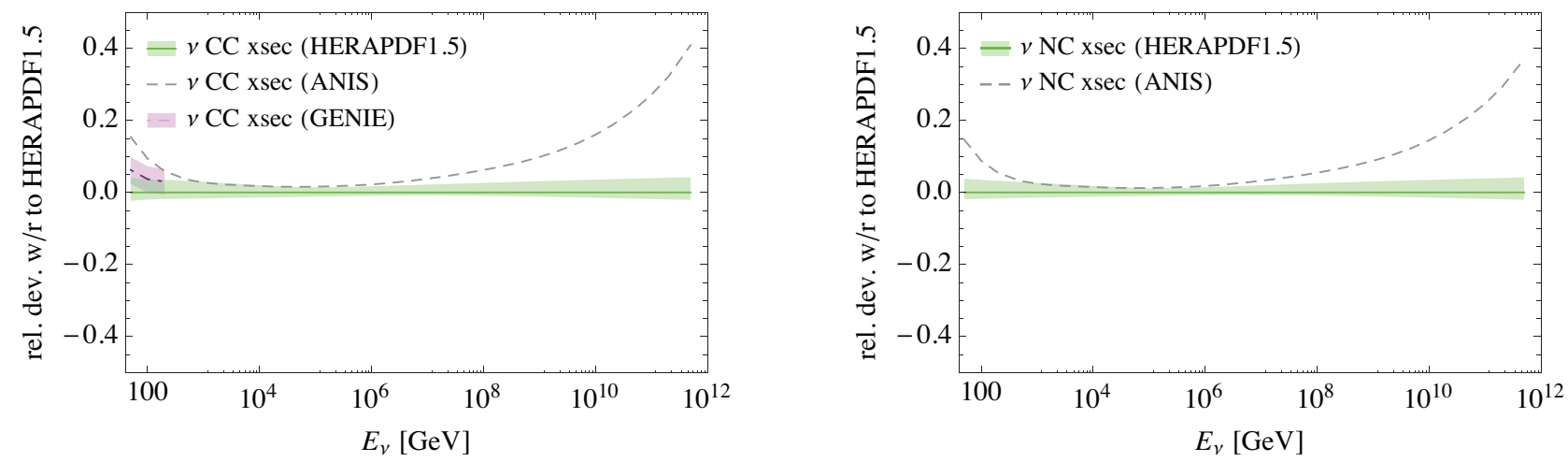

FIG. 12: The relative deviation of the ANIS [57] and GENIE [59] cross-sections from the HERAPDF1.5 central member.
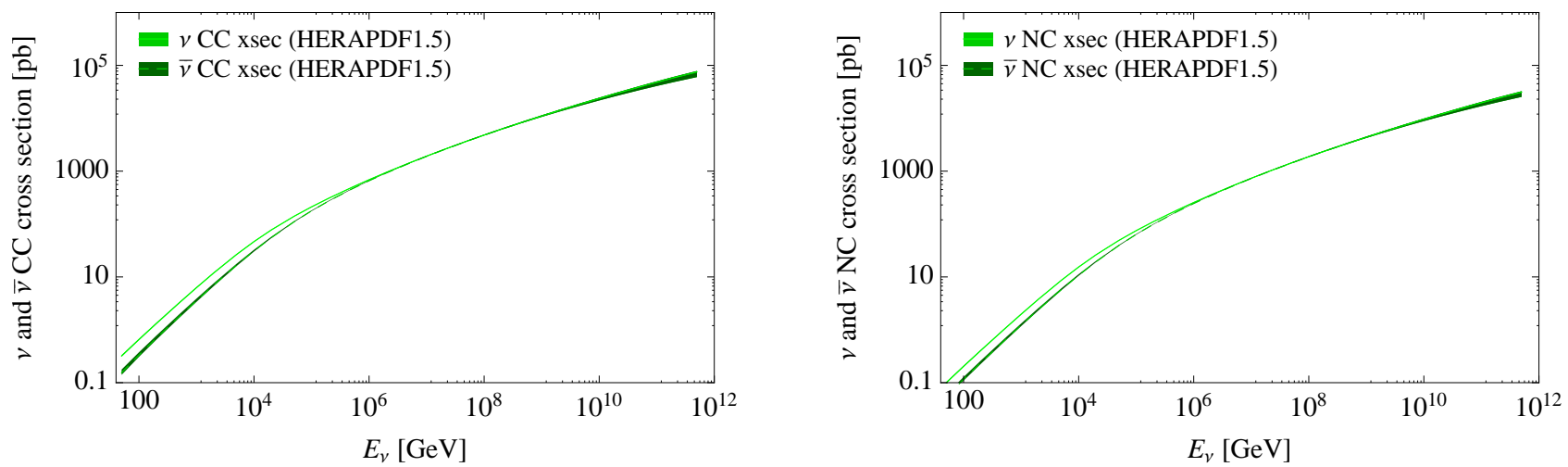

FIG. 13: Neutrino and anti-neutrino cross-sections on isoscalar targets for CC and NC scattering according to HERAPDF1.5.

using HERAPDF1.5 at NLO are shown in Fig. 13. The general trend of the uncertainties can be understood by noting that as one moves to higher neutrino energy one also moves to lower $x$ where the PDF uncertainties are increasing. The PDF uncertainties are smallest at $10^{-2} \lesssim x \lesssim 10^{-1}$, corresponding to $s \sim 10^{5} \mathrm{GeV}^{2}$. Moving to smaller neutrino energies brings us into the high $x$ region where PDF uncertainties increase again. This effect is greater for the HERAPDF1.5 because the HERA data have less statistics at high $x$ than the fixed target data which are included in CT10; however these data have further uncertainties that are not fully accounted for in CT10, e.g. heavy target corrections, deuterium corrections and assumptions regarding higher twist effects. When the high $x$ region becomes important the neutrino and antineutrino cross-sections are different because the valence contribution to $x F_{3}$ is now significant. This is seen in Fig. 13, as is the onset of the linear dependence of the cross-sections for $s<M_{W}^{2}$. Note that our predictions are made for $Q^{2}>1 \mathrm{GeV}^{2}$ since perturbative QCD cannot sensibly be used at lower values. Moreover for $s$ below $\sim 100 \mathrm{GeV}^{2}$, there can be contributions to the cross-section of $\mathcal{O}(10 \%)$ from even lower values of $Q^{2}$ which are not accounted for here; hence we do not show results for $E_{\nu}$ below 50 GeV where there are other contributions to the neutrino cross-section and the use of a code such as GENIE 59 is appropriate. For higher energies, we intend to upgrade ANIS [57] to use the HERAPDF1.5 (differential) cross-sections. Meanwhile we have provided the total DIS cross-sections for CC and NC scattering of neutrinos and antineutrinos on isoscalar targets in Tables I and II and recommend these as a benchmark for use by experimentalists. These cross-sections as well as those for isoscalar targets are available from a webpage [60]; differential cross sections are available upon request. Any measured deviation from these values would signal the need for new physics beyond the DGLAP formalism.

\section{ACKNOWLEDGEMENTS}

We are grateful to James Ferrando for providing us with an up-to-date version of DISPRED and for discussions. We also thank Mike Whalley and Voica Radescu for the speedy implementation of HERAPDF1.5 in LHAPDF. PM and SS thank their colleagues in the Auger and IceCube collaborations for stimulating exchanges and acknowledge partial 
support from the EU Research \& Training Network "Unification in the LHC era" (PITN-GA-2009-237920). 


\begin{tabular}{|c|c|c|c|c|c|c|c|c|}
\hline$E_{\nu}[\mathrm{GeV}]$ & $\sigma_{\mathrm{CC}}[\mathrm{pb}]$ & up & $\begin{array}{c}\text { down } \\
\text { (w/o mem. 9) }\end{array}$ & $\begin{array}{c}\text { down } \\
(\mathrm{w} / \text { mem. 9) } \\
\end{array}$ & $\sigma_{\mathrm{NC}}[\mathrm{pb}]$ & up & $\begin{array}{c}\text { down } \\
\text { (w/o mem. 9) }\end{array}$ & $\begin{array}{c}\text { down } \\
\text { (w/ mem. 9) }\end{array}$ \\
\hline 50 & 0.32 & $4.1 \%$ & $-2.3 \%$ & $-2.4 \%$ & 0.10 & $3.8 \%$ & $-1.9 \%$ & $-2.0 \%$ \\
\hline 100 & 0.65 & $3.8 \%$ & $-2.0 \%$ & $-2.0 \%$ & 0.20 & $3.5 \%$ & $-1.8 \%$ & $-1.8 \%$ \\
\hline 200 & 1.3 & $3.5 \%$ & $-1.8 \%$ & $-1.9 \%$ & 0.41 & $3.2 \%$ & $-1.6 \%$ & $-1.7 \%$ \\
\hline 500 & 3.2 & $3.2 \%$ & $-1.7 \%$ & $-1.8 \%$ & 1.0 & $2.9 \%$ & $-1.5 \%$ & $-1.5 \%$ \\
\hline 1000 & 6.2 & $3.0 \%$ & $-1.6 \%$ & $-1.7 \%$ & 2.0 & $2.7 \%$ & $-1.4 \%$ & $-1.5 \%$ \\
\hline 2000 & 12. & $2.7 \%$ & $-1.6 \%$ & $-1.6 \%$ & 3.8 & $2.4 \%$ & $-1.3 \%$ & $-1.4 \%$ \\
\hline 5000 & 27. & $2.3 \%$ & $-1.5 \%$ & $-1.5 \%$ & 8.6 & $2.1 \%$ & $-1.3 \%$ & $-1.3 \%$ \\
\hline 10000 & 47. & $2.0 \%$ & $-1.4 \%$ & $-1.4 \%$ & 15. & $1.8 \%$ & $-1.2 \%$ & $-1.2 \%$ \\
\hline 20000 & 77. & $1.8 \%$ & $-1.3 \%$ & $-1.4 \%$ & 26. & $1.6 \%$ & $-1.1 \%$ & $-1.1 \%$ \\
\hline 50000 & 140. & $1.5 \%$ & $-1.2 \%$ & $-1.2 \%$ & 49. & $1.3 \%$ & $-1.0 \%$ & $-1.1 \%$ \\
\hline 100000 & 210. & $1.4 \%$ & $-1.2 \%$ & $-1.2 \%$ & 75 & $1.2 \%$ & $-1.0 \%$ & $-1.0 \%$ \\
\hline 200000 & 310. & $1.5 \%$ & $-1.1 \%$ & $-1.1 \%$ & 110. & $1.2 \%$ & $-0.9 \%$ & $-0.9 \%$ \\
\hline 500000 & 490. & $1.6 \%$ & $-1.0 \%$ & $-1.0 \%$ & 180. & $1.3 \%$ & $-0.8 \%$ & $-0.8 \%$ \\
\hline $1 \times 10^{6}$ & 690. & $1.7 \%$ & $-0.9 \%$ & $-0.9 \%$ & 260. & $1.4 \%$ & $-0.8 \%$ & $-0.8 \%$ \\
\hline $2 \times 10^{6}$ & 950. & $1.9 \%$ & $-0.9 \%$ & $-0.9 \%$ & 360. & $1.6 \%$ & $-0.8 \%$ & $-0.8 \%$ \\
\hline $5 \times 10^{6}$ & 1400. & $2.0 \%$ & $-0.9 \%$ & $-0.9 \%$ & 540. & $1.8 \%$ & $-0.8 \%$ & $-0.8 \%$ \\
\hline $1 \times 10^{7}$ & 1900. & $2.2 \%$ & $-0.9 \%$ & $-0.9 \%$ & 730. & $2.0 \%$ & $-0.8 \%$ & $-0.8 \%$ \\
\hline $2 \times 10^{7}$ & 2600 . & $2.3 \%$ & $-0.9 \%$ & $-1.0 \%$ & 980. & $2.2 \%$ & $-0.8 \%$ & $-0.9 \%$ \\
\hline $5 \times 10^{7}$ & 3700 . & $2.5 \%$ & $-0.9 \%$ & $-1.2 \%$ & 1400 . & $2.4 \%$ & $-0.9 \%$ & $-1.1 \%$ \\
\hline $1 \times 10^{8}$ & 4800. & $2.7 \%$ & $-0.9 \%$ & $-1.5 \%$ & 1900. & $2.6 \%$ & $-0.9 \%$ & $-1.3 \%$ \\
\hline $2 \times 10^{8}$ & 6200. & $2.8 \%$ & $-1.0 \%$ & $-2.0 \%$ & 2400 . & $2.7 \%$ & $-1.0 \%$ & $-1.8 \%$ \\
\hline $5 \times 10^{8}$ & 8700. & $3.0 \%$ & $-1.1 \%$ & $-3.0 \%$ & 3400 . & $2.9 \%$ & $-1.0 \%$ & $-2.6 \%$ \\
\hline $1 \times 10^{9}$ & 11000. & $3.1 \%$ & $-1.2 \%$ & $-3.9 \%$ & 4400 . & $3.0 \%$ & $-1.1 \%$ & $-3.4 \%$ \\
\hline $2 \times 10^{9}$ & 14000 . & $3.3 \%$ & $-1.2 \%$ & $-5.0 \%$ & 5600. & $3.2 \%$ & $-1.2 \%$ & $-4.4 \%$ \\
\hline $5 \times 10^{9}$ & 19000. & $3.4 \%$ & $-1.4 \%$ & $-6.8 \%$ & 7600. & $3.4 \%$ & $-1.3 \%$ & $-6.1 \%$ \\
\hline $1 \times 10^{10}$ & 24000 . & $3.6 \%$ & $-1.5 \%$ & $-8.5 \%$ & 9600. & $3.5 \%$ & $-1.4 \%$ & $-7.6 \%$ \\
\hline $2 \times 10^{10}$ & 30000. & $3.7 \%$ & $-1.6 \%$ & $-10.3 \%$ & 12000. & $3.6 \%$ & $-1.5 \%$ & $-9.3 \%$ \\
\hline $5 \times 10^{10}$ & 39000. & $3.8 \%$ & $-1.7 \%$ & $-13.1 \%$ & 16000. & $3.8 \%$ & $-1.7 \%$ & $-11.8 \%$ \\
\hline $1 \times 10^{11}$ & 48000. & $4.0 \%$ & $-1.8 \%$ & $-15.2 \%$ & 20000. & $3.9 \%$ & $-1.8 \%$ & $-13.9 \%$ \\
\hline $2 \times 10^{11}$ & 59000. & $4.1 \%$ & $-1.9 \%$ & $-17.5 \%$ & 24000 . & $4.0 \%$ & $-1.9 \%$ & $-16.1 \%$ \\
\hline $5 \times 10^{11}$ & 75000 . & $4.2 \%$ & $-2.0 \%$ & $-20.3 \%$ & 31000. & $4.2 \%$ & $-2.0 \%$ & $-18.8 \%$ \\
\hline
\end{tabular}

TABLE I: Neutrino CC and NC cross-sections on isoscalar targets, along with their uncertainties, in the perturbative DGLAP formalism at NLO, using HERAPDF1.5 (both with and without member 9). 


\begin{tabular}{|c|c|c|c|c|c|c|c|c|}
\hline$E_{\nu}[\mathrm{GeV}]$ & $\sigma_{\mathrm{CC}}[\mathrm{pb}]$ & up & $\begin{array}{c}\text { down } \\
\text { (w/o mem. 9) }\end{array}$ & $\begin{array}{c}\text { down } \\
\text { (w/ mem. 9) } \\
\end{array}$ & $\sigma_{\mathrm{NC}}[\mathrm{pb}]$ & up & $\begin{array}{c}\text { down } \\
\text { (w/o mem. 9) }\end{array}$ & $\begin{array}{c}\text { down } \\
\text { (w/ mem. 9) }\end{array}$ \\
\hline 50 & 0.15 & $15.0 \%$ & $-9.0 \%$ & $-9.0 \%$ & 0.05 & $12.0 \%$ & $-6.4 \%$ & $-6.4 \%$ \\
\hline 100 & 0.33 & $13.3 \%$ & $-7.4 \%$ & $-7.4 \%$ & 0.12 & $10.7 \%$ & $-5.7 \%$ & $-5.7 \%$ \\
\hline 200 & 0.69 & $11.9 \%$ & $-6.5 \%$ & $-6.5 \%$ & 0.24 & $9.6 \%$ & $-5.1 \%$ & $-5.1 \%$ \\
\hline 500 & 1.8 & $10.5 \%$ & $-5.7 \%$ & $-5.7 \%$ & 0.61 & $8.6 \%$ & $-4.6 \%$ & $-4.6 \%$ \\
\hline 1000 & 3.6 & $9.4 \%$ & $-5.2 \%$ & $-5.2 \%$ & 1.20 & $7.8 \%$ & $-4.2 \%$ & $-4.2 \%$ \\
\hline 2000 & 7. & $8.3 \%$ & $-4.6 \%$ & $-4.6 \%$ & 2.4 & $7.0 \%$ & $-3.8 \%$ & $-3.8 \%$ \\
\hline 5000 & 17. & $6.5 \%$ & $-3.7 \%$ & $-3.7 \%$ & 5.8 & $5.7 \%$ & $-3.2 \%$ & $-3.2 \%$ \\
\hline 10000 & 31. & $5.1 \%$ & $-3.0 \%$ & $-3.0 \%$ & 11. & $4.6 \%$ & $-2.7 \%$ & $-2.7 \%$ \\
\hline 20000 & 55. & $3.8 \%$ & $-2.3 \%$ & $-2.3 \%$ & 19. & $3.6 \%$ & $-2.1 \%$ & $-2.1 \%$ \\
\hline 50000 & 110. & $2.5 \%$ & $-1.7 \%$ & $-1.7 \%$ & 39. & $2.4 \%$ & $-1.5 \%$ & $-1.5 \%$ \\
\hline 100000 & 180. & $1.9 \%$ & $-1.4 \%$ & $-1.4 \%$ & 64. & $1.7 \%$ & $-1.2 \%$ & $-1.2 \%$ \\
\hline 200000 & 270 & $1.7 \%$ & $-1.2 \%$ & $-1.2 \%$ & 99. & $1.4 \%$ & $-1.0 \%$ & $-1.0 \%$ \\
\hline 500000 & 460. & $1.7 \%$ & $-1.1 \%$ & $-1.1 \%$ & 170. & $1.4 \%$ & $-0.9 \%$ & $-0.9 \%$ \\
\hline $1 \times 10^{6}$ & 660. & $1.8 \%$ & $-1.0 \%$ & $-1.0 \%$ & 240. & $1.5 \%$ & $-0.8 \%$ & $-0.8 \%$ \\
\hline $2 \times 10^{6}$ & 920. & $1.9 \%$ & $-1.0 \%$ & $-1.0 \%$ & 350. & $1.6 \%$ & $-0.8 \%$ & $-0.8 \%$ \\
\hline $5 \times 10^{6}$ & 1400. & $2.1 \%$ & $-0.9 \%$ & $-0.9 \%$ & 530. & $1.9 \%$ & $-0.8 \%$ & $-0.8 \%$ \\
\hline $1 \times 10^{7}$ & 1900. & $2.2 \%$ & $-0.9 \%$ & $-0.9 \%$ & 730. & $2.0 \%$ & $-0.8 \%$ & $-0.8 \%$ \\
\hline $2 \times 10^{7}$ & 2500. & $2.3 \%$ & $-0.9 \%$ & $-1.0 \%$ & 980. & $2.2 \%$ & $-0.8 \%$ & $-0.9 \%$ \\
\hline $5 \times 10^{7}$ & 3700 . & $2.5 \%$ & $-0.9 \%$ & $-1.2 \%$ & 1400. & $2.4 \%$ & $-0.9 \%$ & $-1.1 \%$ \\
\hline $1 \times 10^{8}$ & 4800. & $2.7 \%$ & $-1.0 \%$ & $-1.5 \%$ & 1900. & $2.6 \%$ & $-0.9 \%$ & $-1.3 \%$ \\
\hline $2 \times 10^{8}$ & 6200. & $2.8 \%$ & $-1.0 \%$ & $-2.0 \%$ & 2400 . & $2.7 \%$ & $-1.0 \%$ & $-1.8 \%$ \\
\hline $5 \times 10^{8}$ & 8700. & $3.0 \%$ & $-1.1 \%$ & $-3.0 \%$ & 3400 . & $2.9 \%$ & $-1.0 \%$ & $-2.6 \%$ \\
\hline $1 \times 10^{9}$ & 11000. & $3.1 \%$ & $-1.2 \%$ & $-3.9 \%$ & 4400. & $3.0 \%$ & $-1.1 \%$ & $-3.4 \%$ \\
\hline $2 \times 10^{9}$ & 14000 . & $3.3 \%$ & $-1.2 \%$ & $-5.0 \%$ & 5600. & $3.2 \%$ & $-1.2 \%$ & $-4.4 \%$ \\
\hline $5 \times 10^{9}$ & 19000. & $3.4 \%$ & $-1.4 \%$ & $-6.8 \%$ & 7600 & $3.4 \%$ & $-1.3 \%$ & $-6.1 \%$ \\
\hline $1 \times 10^{10}$ & 24000 . & $3.6 \%$ & $-1.5 \%$ & $-8.5 \%$ & 9600. & $3.5 \%$ & $-1.4 \%$ & $-7.6 \%$ \\
\hline $2 \times 10^{10}$ & 30000. & $3.7 \%$ & $-1.6 \%$ & $-10.3 \%$ & 12000. & $3.6 \%$ & $-1.5 \%$ & $-9.3 \%$ \\
\hline $5 \times 10^{10}$ & 39000. & $3.8 \%$ & $-1.7 \%$ & $-13.1 \%$ & 16000. & $3.8 \%$ & $-1.7 \%$ & $-11.8 \%$ \\
\hline $1 \times 10^{11}$ & 48000. & $4.0 \%$ & $-1.8 \%$ & $-15.2 \%$ & 20000. & $3.9 \%$ & $-1.8 \%$ & $-13.9 \%$ \\
\hline $2 \times 10^{11}$ & 59000 . & $4.1 \%$ & $-1.9 \%$ & $-17.5 \%$ & 24000 . & $4.0 \%$ & $-1.9 \%$ & $-16.1 \%$ \\
\hline $5 \times 10^{11}$ & 75000 . & $4.2 \%$ & $-2.0 \%$ & $-20.3 \%$ & 31000. & $4.2 \%$ & $-2.0 \%$ & $-18.8 \%$ \\
\hline
\end{tabular}

TABLE II: Antineutrino CC and NC cross-sections on isoscalar targets, along with their uncertainties, in the perturbative DGLAP formalism at NLO, using HERAPDF1.5 (both with and without member 9). 
[1] F. D. Aaron et al. [H1 \& ZEUS Collaboration], JHEP 1001 (2010) 109.

[2] P. W. Gorham et al. [ANITA collaboration], Phys. Rev. Lett. 103 (2009) 051103. Phys. Rev. D 82 (2010) 022004.

[3] R. Abbasi et al. [IceCube Collaboration], Phys. Rev. D 82 (2010) 072003; Phys. Rev. D 83 (2011) 092003.

[4] J. Abraham et al. [The Pierre Auger Collaboration], Phys. Rev. Lett. 100 (2008) 211101; Phys. Rev. D 79 (2009) 102001.

[5] I. Kravchenko et al., arXiv:1106.1164 [astro-ph.HE]].

[6] P. Allison et al., arXiv:1105.2854 [astro-ph.IM]].

[7] L. Gerhardt et al., Nucl. Instrum. Meth. A 624 (2010) 85.

[8] Y. Takahashi [ JEM-EUSO Collaboration ], New J. Phys. 11 (2009) 065009.

[9] C. W. James et al., Phys. Rev. D 81 (2010) 042003.

[10] G. Altarelli, G. Parisi, Nucl. Phys. B 126 (1977) 298.

[11] V. N. Gribov, L. N. Lipatov, Sov. J. Nucl. Phys. 15 (1972) 438.

[12] L. N. Lipatov, Sov. J. Nucl. Phys. 20 (1975) 94.

[13] Y. L. Dokshitzer, Sov. Phys. JETP 46 (1977) 641.

[14] E. A. Kuraev, L. N. Lipatov, V. S. Fadin, Sov. Phys. JETP 45 (1977) 199.

[15] I. I. Balitsky, L. N. Lipatov, Sov. J. Nucl. Phys. 28 (1978) 822.

[16] L. N. Lipatov, Sov. Phys. JETP 63 (1986) 904.

[17] G. Altarelli, R. D. Ball, S. Forte, Nucl. Phys. B 742 (2006) 1.

[18] M. Ciafaloni, D. Colferai, G. P. Salam, A. M. Stasto, Phys. Lett. B 635 (2006) 320.

[19] C. D. White, R. S. Thorne, Phys. Rev. D 74 (2006) 014002.

[20] J. Rojo, G. Altarelli, R. D. Ball, S. Forte, arXiv:0907.0443 [hep-ph]].

[21] F. Caola, S. Forte, J. Rojo, Phys. Lett. B686 (2010) 127; Nucl. Phys. A854 (2011) 32.

[22] F. Gelis, E. Iancu, J. Jalilian-Marian, R. Venugopalan, Ann. Rev. Nucl. Part. Sci. 60 (2010) 463.

[23] V. P. Goncalves, P. Hepp, Phys. Rev. D 83 (2011) 014014

[24] R. Gandhi, C. Quigg, M. H. Reno, I. Sarcevic, Phys. Rev. D 58 (1998) 093009.

[25] S. Chekanov et al. [ZEUS Collaboration], Phys. Rev. D 67 (2003) 012007.

[26] A. Cooper-Sarkar, S. Sarkar, JHEP 0801 (2008) 075.

[27] A. Connolly, R. S. Thorne, D. Waters, arXiv:1102.0691 [hep-ph].

[28] R. S. Thorne, R. G. Roberts, Phys. Rev. D 57 (1998) 6871.

[29] R. S. Thorne, Phys. Rev. D 73 (2006) 054019.

[30] L. A. Anchordoqui, A. M. Cooper-Sarkar, D. Hooper, S. Sarkar, Phys. Rev. D 74 (2006) 043008.

[31] A. Kusenko, T. J. Weiler, Phys. Rev. Lett. 88 (2002) 161101.

[32] L. Anchordoqui, T. Han, D. Hooper, S. Sarkar, Astropart. Phys. 25 (2006) 14.

[33] S. Palomares-Ruiz, A. Irimia, T. J. Weiler, Phys. Rev. D 73 (2006) 083003.

[34] L. A. Anchordoqui, H. Goldberg, D. Gora, T. Paul, M. Roth, S. Sarkar, L. L. Winders, Phys. Rev. D 82 (2010) 043001.

[35] L. A. Anchordoqui, T. Montaruli, Ann. Rev. Nucl. Part. Sci. 60 (2010) 129.

[36] R. Devenish, A. Cooper-Sarkar, "Deep inelastic scattering", Oxford University Press (2004).

[37] S. Alekhin et al., arXiv:1101.0536 [hep-ph].

[38] R. D. Ball, L. Del Debbio, S. Forte, A. Guffanti, J. I. Latorre, J. Rojo, M. Ubiali, Nucl. Phys. B838 (2010) 136-206. arXiv:1002.4407 [hep-ph]].

[39] W. K. Tung, H. L. Lai, A. Belyaev, J. Pumplin, D. Stump, C. P. Yuan, JHEP 0702 (2007) 053.

[40] H. -L. Lai, M. Guzzi, J. Huston, Z. Li, P. M. Nadolsky, J. Pumplin, C. -P. Yuan, Phys. Rev. D82 (2010) 074024.

[41] A. Cooper-Sarkar [on behalf of the H1 \& ZEUS Collaborations], arXiv:1012.1438 [hep-ph].

[42] A. Accardi, W. Melnitchouk, J. F. Owens, M. E. Christy, C. E. Keppel, L. Zhu, J. G. Morfin, arXiv:1102.3686 [hep-ph].

[43] A. M. Cooper-Sarkar, J. Phys. G 28 (2002) 2669.

[44] M. Kramer, 1, F. I. Olness, D. E. Soper, Phys. Rev. D62 (2000) 096007.

[45] J. M. Campbell, J. W. Huston, W. J. Stirling, Rept. Prog. Phys. 70 (2007) 89.

[46] M. Botje et al., arXiv:1101.0538 [hep-ph].

[47] J. Ferrando, arXiv:1007.5489 [hep-ph]]; J. Ferrando, P. Mertsch, in preparation.

[48] M. Botje, Eur. Phys. J. C 14 (2000) 285.

[49] M. Galassi et al., "GNU Scientific Library Reference Manual" (3rd Ed.), ISBN 0954612078.

[50] http://projects.hepforge.org/lhapdf/pdfsets

[51] http://www.phys.epsu.edu/ cteq/\#PDFs

[52] http://projects.hepforge.org/mstwpdf/

[53] P. Nadolsky, private communication.

[54] R. Fiore, L. L. Jenkovszky, A. V. Kotikov, F. Paccanoni, A. Papa, Phys. Rev. D 73 (2006) 053012.

[55] M. M. Block, P. Ha, D. W. McKay, Phys. Rev. D82 (2010) 077302.

[56] A. Y. Illarionov, B. A. Kniehl, A. V. Kotikov, arXiv:1105.2829 [hep-ph]].

[57] A. Gazizov, M. P. Kowalski, Comput. Phys. Commun. 172 (2005) 203.

[58] R. Abbasi et al. [ IceCube Collaboration ], Phys. Rev. D83 (2011) 012001.

[59] C. Andreopoulos et al., Nucl. Instrum. Meth. A614 (2010) 87.

[60] http://www-pnp.physics.ox.ac.uk/ cooper/neutrino/ 